\title{
A Tabu Search Algorithm for Sparse Placement of Wavelength Converters in Optical Networks ${ }^{\star}$
}

\author{
Namik Sengezer and Ezhan Karasan \\ Electrical and Electronics Engineering Dept., Bilkent University, 06800 Bilkent, \\ Ankara, Turkey \\ \{namik, ezhan\}@ee.bilkent.edu.tr
}

\begin{abstract}
In this paper, we study the problem of placing limited number of wavelength converting nodes in a multi-fiber network with static traffic demands and propose a tabu search based heuristic algorithm. The objective of the algorithm is to achieve the performance of full wavelength conversion in terms of minimizing the total number of fibers used in the network by placing minimum number of wavelength converting nodes. We also present a greedy algorithm and compare its performance with the tabu search algorithm. Finally, we present numerical results that demonstrate the high correlation between placing a wavelength converting node and the amount of transit traffic passing through that node.
\end{abstract}

\section{Introduction}

All optical WDM networks, providing extremely large bandwidths, is one of the most promising solutions for the increasing need for high-speed data transport. In all optical networks, data is transmitted solely in the optical domain from source to destination without being converted into the electronic form [1]. This is achieved with the use of optical cross-connects (OXCs) which can optically switch the data from an incoming fiber to an outgoing fiber. There are two types of OXCs: wavelength-selective cross-connects (WSXCs) and wavelengthinterchanging cross-connects (WIXCs). Any signal passing through a WSXC should occupy the same wavelength on the incoming and outgoing fibers. In WIXCs, the wavelength of the signal can be changed during switching [2].

Transmissions between nodes are done by establishing lightpaths that are direct optical connections between their source and destination nodes. In a wavelength selective network, which is comprised of WSXCs only, the same wavelength must be used on all the links along a lightpath. This is called the wavelength continuity constraint. This constraint can be removed by using WIXCs. The high cost of WIXCs leads to the idea of optical networks with sparse wavelength conversion where only a limited number of nodes in the network are equipped with WIXCs [3-9].

\footnotetext{
* This work was partially supported by the EU-FP6 IST-NoE e-Photon/ONe and by The Scientific and Technical Research Council of Turkey (TUBITAK) under projects EEEAG-101E048 and EEAG-199E005.
} 
In sparse wavelength conversion, the main objective is to locate the limited number of WIXCs to achieve the optimum performance. It is shown that the problem of finding the locations of the minimum number of WIXCs so that no more wavelengths are needed than if there were WIXCs at all nodes is NPcomplete [10].

The performance measures considered in the literature change with the type of traffic. In the dynamic traffic case, the overall blocking probability and in the static traffic case the number of WIXCs or the total cost of the network are generally used as performance measures. There are a number of studies in the literature focusing on each of these cases. In [3] and [4], the paths are divided into segments by WIXCs, and it is shown that the end-to-end blocking probability of a path is minimized when each segment has equal blocking probability. The optimal placement of WIXCs minimizing the overall network blocking probability for arbitrary network topologies is obtained by using an exhaustive search method employing auxiliary graphs in [5]. The relationship between the Routing and Wavelength Assignment (RWA) algorithms and the WIXC placement algorithm is investigated in [6], and two different WIXC placement heuristics minimizing the overall blocking probability are proposed. An optimization model for WIXC placement is presented in [7] based on the formulation of the end-to-end success probability between a pair of nodes as a function of WIXC locations. The issue of designing transparent optical networks minimizing the network cost while establishing all the lightpaths is studied in [8], and three heuristics are proposed for wavelength assignment and WIXC placement. A scalable solution technique for optimum placement of WIXCs is presented in [9]. The overall problem is modelled by a sequence of Integer Linear Programming (ILP) formulations with the objective of minimizing the number of WIXCs necessary to achieve the minimum fiber usage in the network.

In this paper, we assume static traffic demands in a multi-fiber network with fixed number of wavelengths on each fiber. The main objective is to place the minimum number of WIXCs necessary for achieving the minimum fiber usage which is obtained in a network having full wavelength conversion capability. We utilize an ILP based solution using path formulation for the routing problem and an iterative heuristic for the wavelength assignment. For the WIXC placement, we propose a tabu search based algorithm. This algorithm can find solutions in a reasonable amount of time and can be used in conjunction with different routing and wavelength assignment algorithms. We also implement a greedy search algorithm for WIXC placement and use its performance as a basis for comparison with the tabu search algorithm.

The remainder of the paper is organized as follows. In Section 2, the routing and wavelength assignment algorithms used in this study are presented. The greedy and tabu search algorithms proposed for the WIXC placement problem are introduced in Section 3. Section 4 presents some numerical results and the performance comparison of these algorithms. Finally, the conclusions are discussed in Section 5. 


\section{Routing and Wavelength Assignment}

Although the routing and wavelength assignment problems can be solved jointly, the two subproblems are decoupled in this paper in order to reduce the computational complexity of the overall problem.

\subsection{Routing Problem}

The final objective in this work is to use the same number of fibers obtained with the full wavelength conversion while WIXCs are placed only at selected nodes. Concluding from this point of view, the routes to be used in conjunction with the sparse conversion network are obtained considering full wavelength conversion.

The network is represented by an undirected graph where the links and the connections are bidirectional. The set of $k$ shortest paths between all node pairs is given by $P$. Let $Z$ denote the set of all node pairs and $L$ represent the set of links. For a node pair $z=(m, n) \in \mathrm{Z}, d_{z}$ denotes the number of lightpath requests between nodes $m$ and $n$. The decision variable $f_{l}$ denotes the number of fibers on link $l$ and the routing variable $X_{p z}$ represents the number of lightpaths for node pair $z$ that are assigned to path $p$. The number of wavelengths on each fiber is denoted by $W$. The link-path incidence matrix is given by $J=\left[j_{l p}\right]$, where

$$
j_{l p}= \begin{cases}1 & \text { if link } l \text { is on path } p, \\ 0 & \text { otherwise }\end{cases}
$$

The ILP formulation for the routing problem which minimizes the total number of fibers with full conversion is given by

$$
\text { Minimize } \sum_{l \in L} f_{l}
$$

Subject to

$$
\begin{gathered}
\sum_{p \in P} X_{p z}=d_{z}, \forall z \in Z, d_{z} \in D \quad \text { (demand constraints) } \\
\sum_{z \in Z} \sum_{p \in P} X_{p z} j_{l p} \leq W \times f_{l}, \forall l \in L \quad \text { (capacity constraints) } \\
f_{l} \in Z^{+}, \forall l \in L \\
X_{p z} \in Z^{+}, \forall z \in Z, \forall p \in P
\end{gathered}
$$

The set of routing variables $\left\{X_{p z}\right\}$ in the optimum solution indicates paths used by the lightpaths. 


\subsection{Wavelength Assignment Problem}

Next, we consider the wavelength assignment problem in optical networks with sparse wavelength conversion. For the wavelength assignment subproblem, an iterative heuristic algorithm based on the longest path first approach is used. For a given lightpath, each segment corresponds to the portion of the lightpath lying between two subsequent WIXCs along that lightpath. For the full wavelength conversion case in Section 2.1, segments correspond to individual links. All lightpaths obtained as the solution of the routing problem are divided into segments. These segments are sorted according to their lengths in a descending order. Starting from top of the list, the first available wavelength is assigned to each segment. When no available wavelength is found along a segment, that segment is placed at the top of the list, and all wavelength assignments are done from the beginning. This procedure is repeated for a maximum number of iterations, and when there is no available wavelength along a segment, the wavelength which is unavailable on the minimum number of links along that segment is determined, and the number of fibers on these links are incremented.

The pseudo-code for this algorithm is given below. The maximum number of times the list is reordered is represented by the variable iteration_limit.

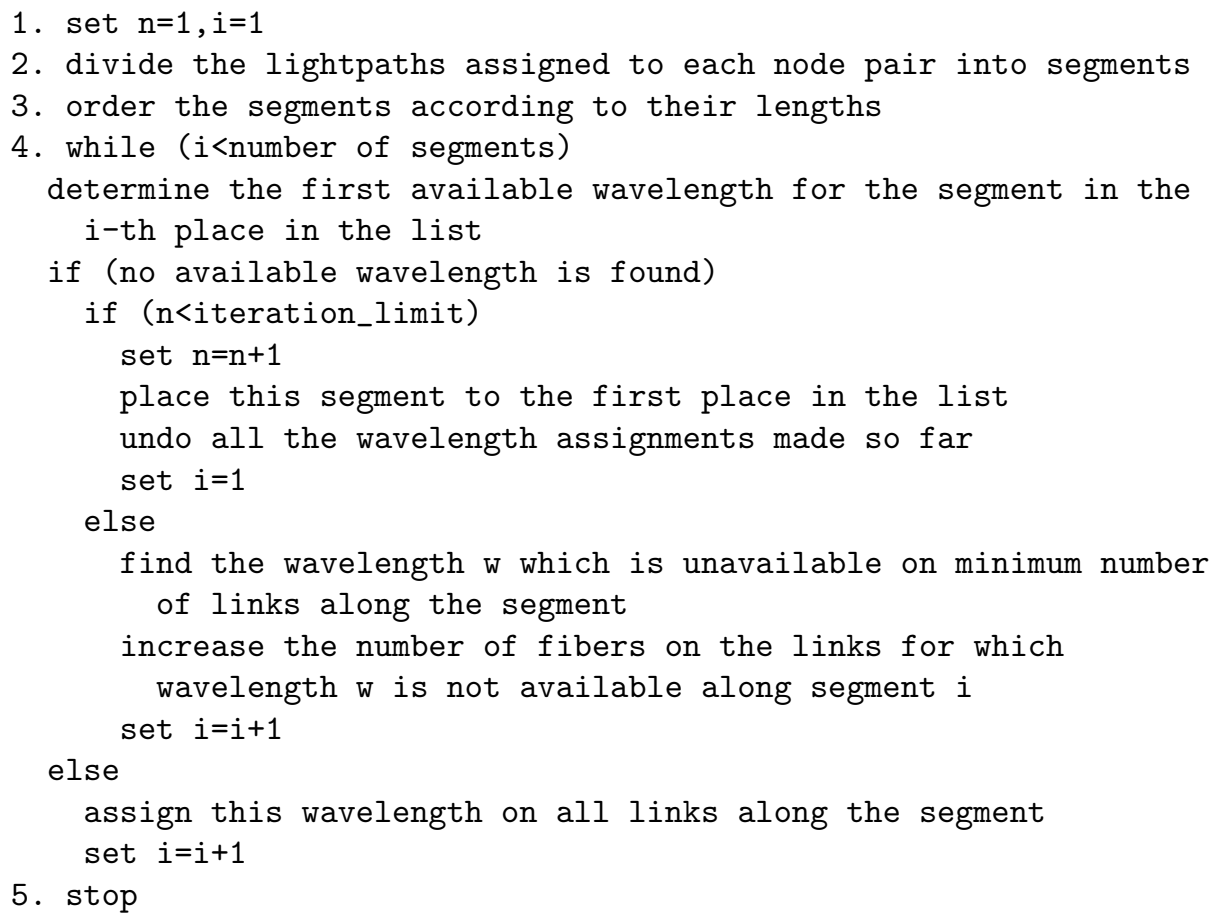

\section{WIXC Placement}

Our objective is to minimize the number of WIXCs in the network necessary to satisfy all the lightpath requests with the same total number of fibers as in 
the case of full wavelength conversion and to find the optimal locations for these WIXCs. To this end, we propose a tabu search algorithm and also implement a greedy search algorithm for comparison purposes that is used as the initial solution for the tabu search algorithm.

\subsection{Greedy Search (GS) Algorithm}

The target minimum number of fibers and the routes of the lightpaths are calculated beforehand using the ILP formulation described in Section 2 assuming full conversion. The flowchart of the GS algorithm is shown in Figure 1. The algorithm starts with no WIXCs. Each move in the algorithm consists of adding a WIXC to one of the nodes with no WIXCs. The node at which placing the WIXC reduces the total number of fibers most is determined. If there are multiple such nodes, the node to place next WIXC is chosen randomly among such nodes. When the target minimum number of fibers is reached, the algorithm stops. In order to determine the number of fibers on each link for each WIXC placement configuration, the wavelength assignment algorithm described in Section 2 is used. In our numerical studies, GS algorithm is executed a number of times in order to generate multiple solutions and the best solution is reported.

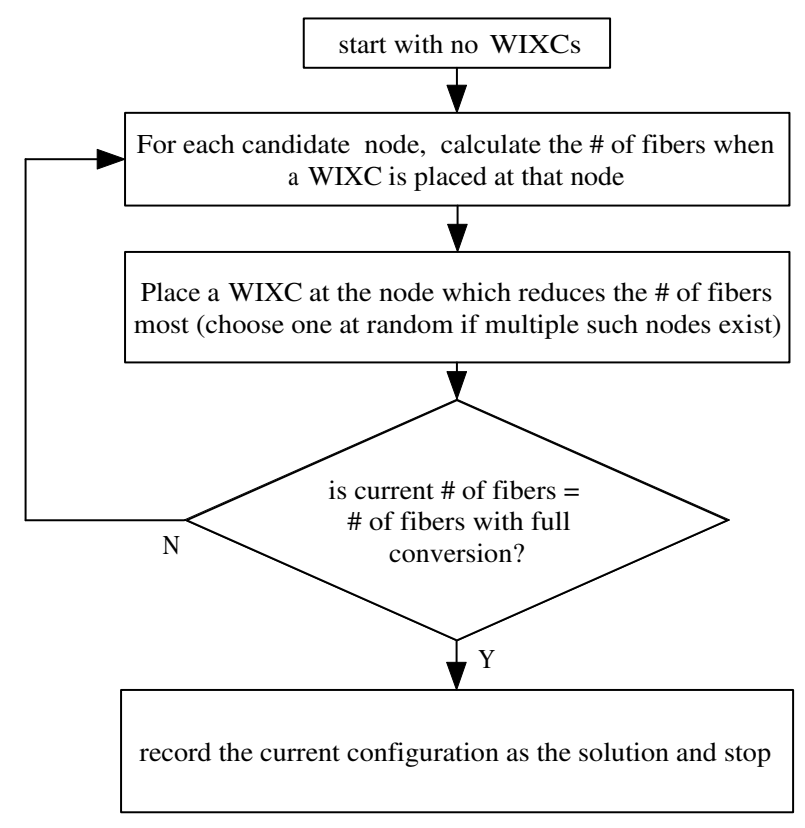

Fig. 1. Flowchart of the GS algorithm 


\subsection{Tabu Search (TS) Algorithm}

Tabu search is an iterative improvement procedure which starts from an initial solution and gradually improves the objective function by a series of moves [12]. Its main difference from other iterative search techniques is that it allows non-improving moves to escape from local optima. Previously made moves are forbidden for a number of iterations by declaring these moves as tabu in order to avoid cycles.

The search space consists of all possible WIXC placements achieving the target number of fibers. The objective function is the number of nodes where a WIXC is placed. We consider three types of possible moves: addition of a WIXC to one of the nodes (add move), dropping one of the WIXCs (drop move) and adding a WIXC to a node while dropping another at the same time (exchange move).

The initial solution for the TS algorithm can be the full conversion case (since the minimum number of fibers is attainable by full conversion) or one of the solutions generated by the GS algorithm. The best solution generated by the GS algorithm is used as the initial solution for TS algorithm in this paper.

At each step, the list of all possible moves is calculated. If there exist possible drop moves, next move is chosen randomly among them. Otherwise, if possible, the next move is chosen among the exchange moves. If neither a drop nor an exchange move is possible, the next move is chosen among the possible add moves. Whenever a move is made, the move together with the existing configuration of WIXCs is added to the tabu list. The tenure of each entry in the tabu list is chosen randomly. The best solution, which is the configuration with the minimum number of WIXCs found so far, is stored. There are two stopping criteria for the algorithm: the conditions of no possible non-tabu moves and no improvement in the objective function for a number of iterations which is denoted by the parameter no_imp_limit.

With this state of the TS algorithm described so far, when a solution is found the algorithm has a tendency to return to that solution after a number of iterations since drop moves always have a higher priority than others. In order to overcome this problem and find other solutions that are not in the close neighborhood, a diversification step is introduced so that unvisited regions of the solution space are also visited. This diversification step is executed when no improvement in the objective function is achieved for a certain number of iterations denoted by the parameter diverse_start. In the diversification step, the drop and exchange moves are not considered for a number of iterations, represented by diversification_limit, leading to an escape from the local minima. The flowchart of the TS algorithm is given in Figure 2.

\section{Numerical Results}

The WIXC placement algorithms are tested using the 19-node European Optical Network (EON) [11]. The experiments are performed for different number of 


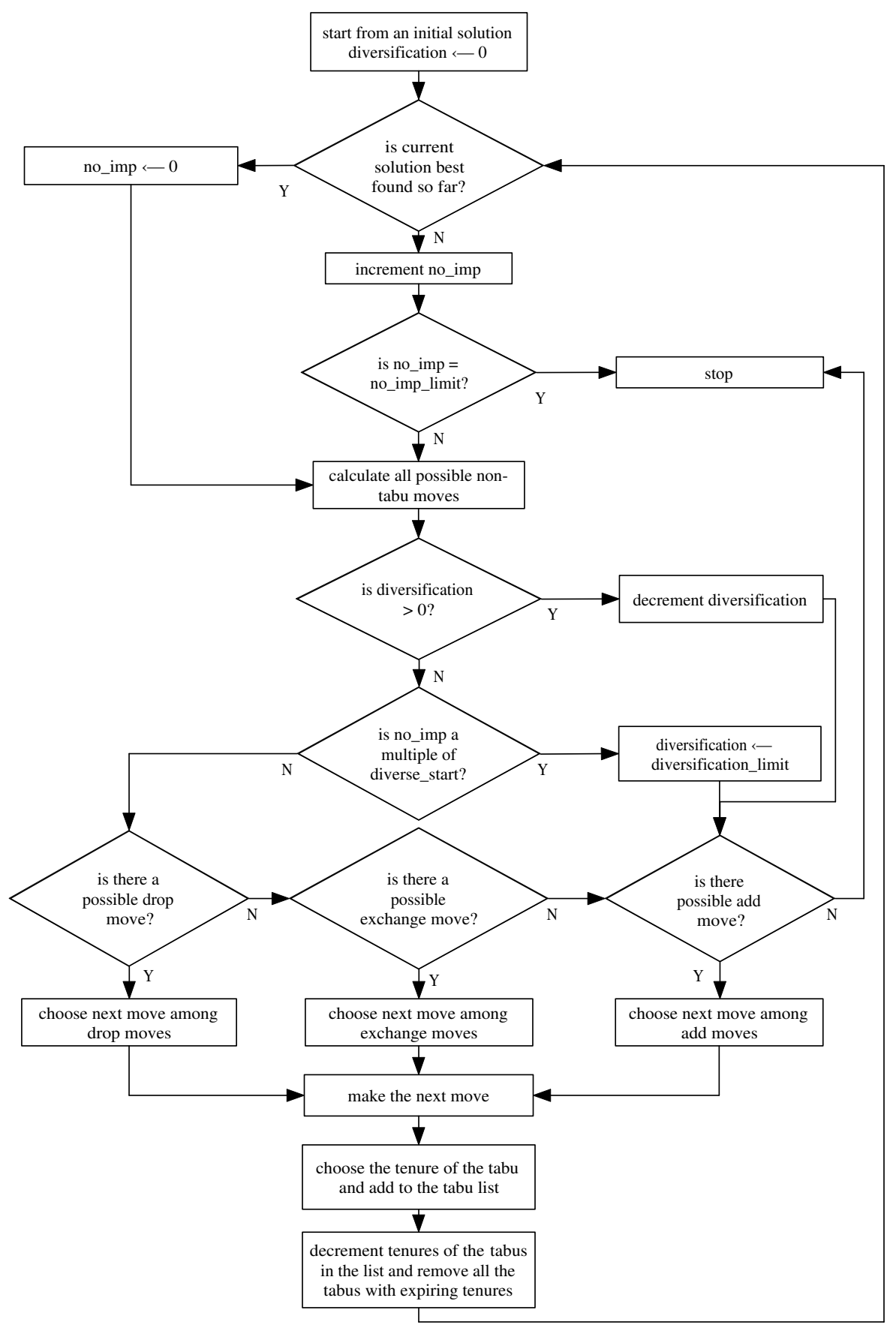

Fig. 2. Flowchart of the TS algorithm 
wavelengths per fiber: $W=16,32$ and 64 . For each value of $W, 50$ different traffic demand matrices are generated, and the average values over these runs are reported. In the traffic model used in this study, lightpath demands exist between each pair of nodes. The number of demands between each node pair is randomly selected between [1-3], [2-6] and [4-12] for 16, 32 and 64 wavelengths, respectively. First, the GS algorithm is run a number of times in order to provide multiple solutions, then the TS algorithm is run starting from the best solution generated by the GS algorithm as initial the solution. The following values are used for the parameters in the TS algorithm: no_imp_limit $=100$, diverse_start $=25$, diversification_limit $=10$. The tenure time of each entry in tabu tabu list is chosen at random between 20 and 40 iterations.

The average number of nodes with WIXCs in the best solutions found by the GS and TS algorithms are reported in Table 1. The TS algorithm improves the best solution found by the GS algorithm in approximately $6 \%$ of the trials for 16 wavelengths, in $12 \%$ for 32 wavelengths and in $22 \%$ for 64 wavelengths. For small $W$, full conversion performance can be achieved with less number of WIXCs and it is more likely that the GS algorithm gives the same number of WIXCs as the TS algorithm. As $W$ increases, it becomes more likely that the TS algorithm improves the results of the GS algorithm. In the cases for which the TS algorithm does not improve the initial solution found by the GS algorithm, it may generate alternative solutions.

Table 1. Average number of WIXCs obtained by the GS and TS algorithms, max. improvement of TS algorithm and percentage of the cases with no improvement

\begin{tabular}{|c|c|c|c|c|}
\hline \multirow{2}{*}{$W$} & \multicolumn{2}{|c|}{$\begin{array}{l}\text { Average \# } \\
\text { of WIXCs }\end{array}$} & $\begin{array}{l}\text { Max improve- } \\
\text { ment in \# of } \\
\text { yynn }\end{array}$ & $\begin{array}{l}\text { percentage of } \\
\text { cases with no } \\
\text { improvement }\end{array}$ \\
\hline 16 & 1.90 & 1.82 & 2 & 94 \\
\hline 32 & 2.38 & 2.22 & 2 & 88 \\
\hline 64 & 2.76 & 2.50 & 2 & 78 \\
\hline
\end{tabular}

The number of WIXCs needed in order to obtain the same number of fibers with the full conversion case increases as $W$ increases: about $9.6 \%$ of all nodes contain WIXCs for $W=16$, and this ratio is $11.7 \%$ for $W=32$ and $13.2 \%$ for $W=64$. The maximum improvement of the TS algorithm in number of WIXCs is 2 for all values of $W$.

The percentage of traffic demand cases for which a WIXC is placed at each node in the best solutions obtained using the TS algorithm is shown in Figure 3. The frequencies of placing WIXCs at certain nodes are higher than the others. Node 9, which has a high nodal degree, is equipped with WIXCs in more than half of the solutions. Nodes 1, 4 and 7 are also have a high tendency to be chosen. The total numbers of demands passing through each node are shown in Figure 4. We observe that there is a high correlation between the number of demands passing through a node and the probability that node is assigned a WIXC in 
the solution given by the TS algorithm. The five nodes that carry the largest number of lightpaths, namely nodes 1, 2, 4, 7 an 9, have the largest likelihood of placing a WIXC. Taking into account that the demands are uniformly distributed with equal parameters for all node pairs, the WIXC placement depends on the network topology and the routing algorithm used.
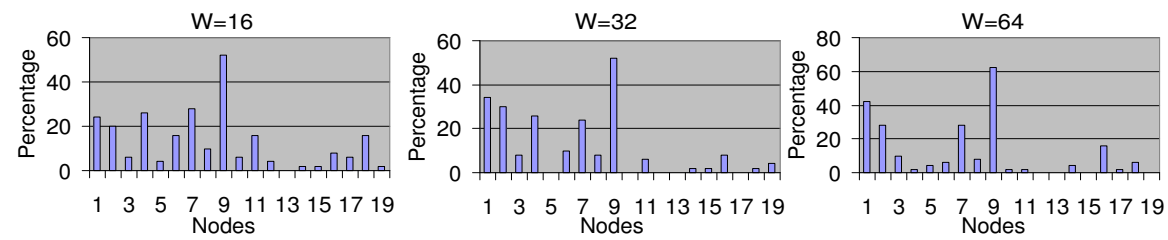

Fig. 3. The distribution of WIXCs at the nodes
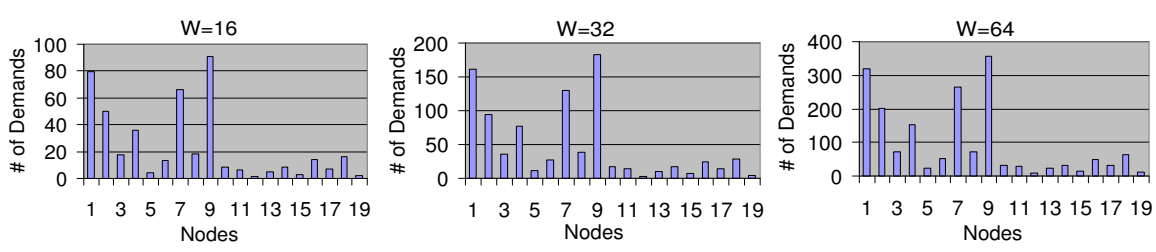

Fig. 4. The number of demands passing through the nodes

\section{Conclusions}

In this paper, a tabu search algorithm for placing limited number of WIXCs in a multi-fiber network under static traffic demands is presented. The objective of the algorithm is to achieve the performance of full wavelength conversion in terms of total number of fibers by placing minimum number of WIXCs. An iterative wavelength assignment algorithm is used in the tabu search algorithm giving satisfactory results in a reasonable amount of time.

The average percentage of nodes with WIXCs to all nodes in the best solution generated by the TS algorithm slightly increases with the number of wavelengths, but it is less than $14 \%$ on the average. We also observe that WIXCs are mostly concentrated at a small number of nodes and there is a significant correlation between the probability that a node is assigned a WIXC in the best solution and the number of demands passing through that node. The distribution of the WIXCs and the number of demands passing through the nodes can be used in 
order to form strategies that may improve the performance of the TS algorithm. One possibility is that certain nodes can be given a higher probability of WIXC assignment while making the movement decisions.

The TS algorithm can be studied further using different RWA algorithms. An important issue to be considered while choosing the wavelength assignment algorithm is the running time which is the determining factor for the overall run time since wavelength assignment is done for every WIXC placement configuration considered for each move of the TS algorithm.

\section{References}

1. R. Ramaswami and K. N. Sivarajan, Optical Networks A Practical Perspective, Morgan Kaufmann Publishers, San Francisco, CA, 1998.

2. E. Karasan and E. Ayanoglu, "Performance of WDM Transport Networks", IEEE Journal on Selected Areas in Communications, 16(7): 1081-1096, Sep. 1998.

3. S. Subramaniam and M. Azizoglu, "On Optimal Converter Placement in Wavelength-Routed Networks", IEEE/ACM Transactions on Networking, 7(5):754-766, Oct. 1999.

4. L. Li and A. K. Somani, "Efficient Algorithms for Wavelength Converter Placement", SPI/Kluwer Optical Networks Magazine, 3(2), April 2002.

5. S. Thiagarajan and A. K. Somani, "An Efficient Algorithm for Optimal Wavelength Converter Placement on Wavelength-Routed Networks with Arbitrary Topologies", Proc. IEEE INFOCOM '99, pp. 916-923, 1999.

6. X. Chu, B. Li, I. Chlamtac, "Wavelength Converter Placement Under Different RWA Algorithms in Wavelength-Routed All-Optical Networks", IEEE Transactions on Communications, 51(4): 607-617, April 2003.

7. S. Gao, X. Jia, C. Huang, D. Du, "An Optimization for Placement of Wavelength Converters to Minimize Blocking Probability in WDM Networks", Journal of Lightwave Technology, 21(3), March 2003.

8. Zymolka, A. Koster, R. Wessaly, "A Transparent Optical Network Design with Sparse Wavelength Conversion", Proc. ONDM 2003, pp. 61-80, 2003.

9. E. Karasan, O. E. Karasan, G. Erdogan, "Optimum Placement of Wavelength Interchanging Nodes in Optical Networks with Sparse Conversion", Proc. of the 9th European Conference on Networks and Optical Communications, Eindhoven, Netherlands, pp. 525-532, 2004.

10. G. Wilfong and P. Winkler, "Ring Routing and Wavelength Translation", Proc. of the 9th Annual ACM-SIAM Symposium on Discrete Algorithms, pp. 333-341, 1998.

11. K. R. Venugopal, M. Shiva Kumar, P. Sreenivasa Kumar, "A heuristic for placement of limited range wavelength converters in all-optical networks", Computer Networks, 35(2-3): 143-163, 2001.

12. Fred Glover and M. Laguna, Tabu Search, Kluwer Academic Publishers, Boston, MA, 1997. 